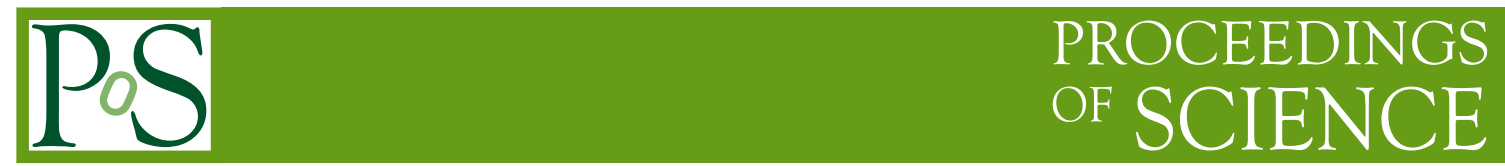

\title{
Review on Bell-Steinberger relations
}

\author{
Giancarlo D'Ambrosio*i \\ INFN-Sezione di Napoli \\ E-mail: gdambrosena.infn.it
}

We illustrate shortly the motivations to search for CPT violation in kaon decays, then we outline the derivation of the Bell-Steinberger relations. Mostly we focus on determination from the BellSteinberger relations of the CPT violating parameters in the kaon system

KAON International Conference

May 21-25 2007

Laboratori Nazionali di Frascati dell'INFN, Rome, Italy

*Speaker.

${ }^{\dagger}$ I thank KLOE and G. Isidori for collaboration 


\section{Introduction}

CPT theorem is based deeply on our understanding of quantum field theory. As we shall see we can test this symmetry to a very meaningful level in the kaon system. Actually to this purpose we will use the Bell Steinberger relations, where unitarity is assumed. We will first introduce briefly CPT theorem and possible violations to motivate a bit the experimental searches, then we will analyze the kaon decays that are involved. Recently thanks to NA48, KTEV, KLOE and CPLEAR the experimental situation is substantially improved.

\section{CPT violation}

Relativistic quantum field theories predict a very important property: CPT invariance [1, 目, 目, \# (see also for notations reviews in [5]), which holds under the following three hypotheses:

- Lorentz invariance

- Hermiticity of the Hamiltonian

- Locality.

CTP-violation and/or the accuracy on which we test CPT is fundamental in physics and searched in several experiments [6]. A theoretical acceptable framework to generate CPT-violation is the one suggested in Ref. [7], where departures from Lorentz invariance generate CPT-even and CPT-odd terms: small non-invariant terms are added to the Standard Model Lagrangian, these are assumed renormalizable (dimension $\leq 4$ ), invariant under $S U(3)_{c} \times S U(2)_{L} \times U(1)_{Y}$ and rotationally and translationally invariant in a preferred frame (then fixed to be the one where the cosmic microwave background is isotropic). String theory is presumably valid up to the Planck scale and argued to be CPT-conserving. But spontaneous CPT-violation is still allowed: S-matrix elements may violate CPT according to the details of the low energy limit. In fact string theory can generate Lorentz and CPT violating terms [8]. Actually there are also explicit quantum field theory examples of spontaneous CPT-violation [9]. Just to give an explicit example of Lorentz violation we mention the one particularly used in cosmic rays and neutrino tests. We change the coefficient of the square of the magnetic field in the Lagrangian of quantum electrodynamics:

$$
\vec{B}^{2} \rightarrow(1+\varepsilon) \vec{B}^{2}
$$

This will cause the velocity of light $c$, given by $c^{2}=1+\varepsilon$ to differ from the maximum velocity of particles, which remains equal to one. This is just one of the terms to be added to the Standard Model Lagrangian [7], invoking an explicit violation of Lorentz invariance.

In principle we could think to challenge CPT by giving up locality but not Lorentz invariance. For instance we could add to the usual Dirac term the following non-local fermionic action [10]

$$
\mathbf{S}=\frac{i \eta}{\pi} \int d^{3} x \int d t d t^{\prime} \bar{\psi}(t, \mathbf{x}) \frac{1}{t-t^{\prime}} \psi\left(t^{\prime}, \mathbf{x}\right)
$$

In order to prove that a CPT lagrangian can generate physical amplitudes it is important to check causality: however it is still disputed if this model produces a satisfactory CPT-violating model [11, 12]. 
I recall, as I will show later, that kaon physics maybe sensitive to Planck scale physics [13, 14]. Bell-Steinberger relations, dictated by the unitarity conditions, are the main tool to improve the CPT kaon physics bounds. Now these bounds are limited by the $K \rightarrow \pi \pi$ measurements, so it is compulsory to improve these experimental results.

\section{CPT in the $K$ 's mass and width matrix}

We can describe mass and decay eigenstates of the kaon system by the diagonalization [1, 2, 15] of the matrices

$$
i \frac{d}{d t}\left[\begin{array}{c}
K^{0} \\
\bar{K}^{0}
\end{array}\right]=\left(\begin{array}{cc}
M_{11}-i \Gamma_{11} / 2 & M_{12}-i \Gamma_{12} / 2 \\
M_{21}-i \Gamma_{21} / 2 & M_{22}-i \Gamma_{22} / 2
\end{array}\right)\left[\begin{array}{c}
K^{0} \\
\bar{K}^{0}
\end{array}\right],
$$

where the relative signs of the matrices $\Gamma$ and $M$ are fixed by the requirement of the correct exponential fall-off of kaon wave function. The eigenvectors are found to be

$$
\begin{aligned}
K_{S, L} & =\frac{\left[\left(1+\varepsilon_{S, L}\right) K^{0}+\left(1-\varepsilon_{S, L}\right) \bar{K}^{0}\right]}{\sqrt{2\left(1+\left|\varepsilon_{S, L}\right|^{2}\right)}} \\
\varepsilon_{S, L} & =\frac{-i \mathfrak{I}\left(M_{12}\right)-\frac{1}{2} \mathfrak{I}\left(\Gamma_{12}\right)}{m_{L}-m_{S}+i\left(\Gamma_{S}-\Gamma_{L}\right) / 2} \mp \Delta \\
& =\varepsilon \mp \Delta
\end{aligned}
$$

where we are encoding in $\Delta$ the CPT violating contributions

$$
\Delta=\frac{\frac{1}{2}\left[M_{11}-M_{22}-\frac{i}{2}\left(\Gamma_{11}-\Gamma_{22}\right)\right]}{m_{L}-m_{S}+i\left(\Gamma_{S}-\Gamma_{L}\right) / 2}
$$

We are using here the convention, $\mathfrak{I}\left(\Gamma_{12}\right)=0$; then we determine the mass CP violation phase

$$
\varepsilon \equiv|\varepsilon| e^{i \varphi_{S W}} \quad \tan \varphi_{S W}=\frac{2\left(m_{L}-m_{S}\right)}{\Gamma_{S}-\Gamma_{L}} .
$$

If we would have used the Wu-Yang phase convention, $\left(\mathfrak{I}\left(A_{0}\right)=0\right)$, we would have obtained the same up to $\mathscr{O}\left(\varepsilon^{\prime} / \varepsilon\right)$.

If CPT is not conserved in $K \rightarrow \pi \pi$ decays, new amplitudes $B_{I}$ 's appear:

$$
\begin{aligned}
& A\left(K^{0} \rightarrow \pi \pi(I)\right) \equiv\left(A_{I}+B_{I}\right) e^{i \delta_{I}} \\
& A\left(\bar{K}^{0} \rightarrow \pi \pi(I)\right) \equiv\left(A_{I}^{*}-B_{I}^{*}\right) e^{i \delta_{I}}
\end{aligned}
$$

Defining as usual

$$
\eta_{+-}=\frac{A\left(K_{L} \rightarrow \pi^{+} \pi^{-}\right)}{A\left(K_{S} \rightarrow \pi^{+} \pi^{-}\right)}=\left|\eta_{+-}\right| e^{i \phi_{+-}} \quad \eta_{00}=\frac{A\left(K_{L} \rightarrow \pi^{0} \pi^{0}\right)}{A\left(K_{S} \rightarrow \pi^{0} \pi^{0}\right)}=\left|\eta_{00}\right| e^{i \phi_{00}}
$$

and noticing that $\varphi_{S W}$ in (2.3) is approximately equal, in the CPT limit, to the phase of $\varepsilon^{\prime}$, then the $\eta$ 's phases must be equal in the CPT limit. In fact the following CPT bound has been established experimentally [5]

$$
\phi_{+-}-\phi_{00}=0.023^{\circ} \pm 0.020^{\circ}
$$




\section{BELL-STEINBERGER RELATIONS}

If we think that the probability conservation is valid up to shorter distances than CPT then even if CPT is violated we can impose that unitarity must be valid [16, 17, 1, 18]. Then if we consider the time evolution of an initial kaon state, $|\Psi\rangle$, which is an arbitrary quantum superposition of the mass and width eigenstates $K_{S}, K_{L}$ :

$$
|\Psi\rangle=a\left|K_{S}\right\rangle+b\left|K_{L}\right\rangle
$$

decaying in a final state $\Gamma$, we have to impose for any $a$ and $b$

$$
\sum_{\Gamma}|\langle\Gamma|T| \Psi\rangle|^{2}=-\frac{d}{d t}|\Psi|^{2}
$$

Terms proportional to $|a|^{2}$ and $|b|^{2}$ lead to the definition of the width

$$
\Gamma_{L}=\sum_{\Gamma} \int d \Gamma\left|\left\langle\Gamma|T| K_{L}\right\rangle\right|^{2} \quad \quad \Gamma_{S}=\sum_{\Gamma} \int d \Gamma\left|\left\langle\Gamma|T| K_{S}\right\rangle\right|^{2}
$$

Mixed terms, proportional to $a b^{*}$ lead to the Bell-Steinberger relation [18] connecting $\mathrm{CP}$ and $\mathrm{CPT}$ violation in the mass matrix to $\mathrm{CP}$ and CPT violation in the decay; in fact neglecting $\mathscr{O}(\varepsilon)$ corrections to the coefficient of the CPT violating parameter, $\delta$, we can write [19]

$$
\left[\frac{\Gamma_{S}+\Gamma_{L}}{\Gamma_{S}-\Gamma_{L}}+i \tan \phi_{\mathrm{SW}}\right]\left[\frac{\Re(\varepsilon)}{1+|\varepsilon|^{2}}-i \mathfrak{I}(\delta)\right]=\frac{1}{\Gamma_{S}-\Gamma_{L}} \sum_{\Gamma}\left\langle\Gamma|T| K_{L}\right\rangle\left\langle\Gamma|T| K_{S}\right\rangle^{*} .
$$

Some considerations are useful

- Historically this relation was used by Bell and Steinberger to put a bound, very close to experiments, to $\varepsilon$ [18]: in fact using Schwartz inequality

$$
\left|\frac{\Gamma_{S}+\Gamma_{L}}{2}-i \Delta M\right| \frac{2 \Re(\varepsilon)}{1+|\varepsilon|^{2}} \leq \sqrt{\Gamma_{L} \Gamma_{S}} \Longrightarrow \frac{2 \Re(\varepsilon)}{1+|\varepsilon|^{2}} \leq 2.9 \times 10^{-2}
$$

where $\Delta M=m_{L}-m_{S}$,

- The advantage of the neutral kaon system is that only a few decay modes give significant contributions to the r.h.s. in (3.1);

- our major quantitative results will be to determine $\Re(\varepsilon)$, without the assumption of CPT invariance, and $\mathfrak{I}(\delta)$, to a very good accuracy. The level of precision will be determined by our experimental knowledge of the right-hand side

- The quantity in square brackets is phase convention independent; this is important since we want to relate our final output to previous determinations with different conventions.

- the sum on the right-hand side involves products of amplitudes, $\sim\left\langle\Gamma|T| K_{L, S}\right\rangle$, and here the interferometry measurements from experiments like KLOE and CPLEAR are very useful; 


\begin{tabular}{c|c|c|c} 
Channel & $B\left(K_{S}\right)$ & $B\left(K_{L}\right)$ & $10^{5} \alpha_{f}^{\mathrm{SM}}$ \\
$\pi^{+} \pi^{-}(\gamma)$ & 0.69 & $2.1 \times 10^{-3}$ & $110.8+105.1 i$ \\
$\pi^{0} \pi^{0}$ & 0.31 & $9.3 \times 10^{-3}$ & $49.2+46.6 i$ \\
& & & \\
$\pi^{ \pm} e^{\mp} \nu$ & $6.7 \times 10^{-4}$ & 0.39 & $0.22+0.00 i$ \\
$\pi^{ \pm} \mu^{\mp} \nu$ & $4.7 \times 10^{-4}$ & 0.27 & $0.17+0.00 i$ \\
& & & \\
$\pi^{0} \pi^{0} \pi^{0}$ & $1.9 \times 10^{-9}$ & 0.21 & $0.06+0.06 i$ \\
$\pi^{+} \pi^{-} \pi^{0}$ & $2.7 \times 10^{-7}$ & 0.12 & $0.04+0.04 i$ \\
$\pi^{+} \pi^{-} \gamma_{\mathrm{DE}}$ & $10^{-5}$ & $10^{-5}$ & $<0.01$
\end{tabular}

Table 1: Actual SM expectations for Bell Steinberger relations

actually to this purpose we divide the final states, $\Gamma$ 's, into semileptonic and hadronic states. For hadronic states,

$$
\alpha_{i} \equiv \frac{1}{\Gamma_{S}}\left\langle\mathscr{A}_{L}(i) \mathscr{A}_{S}^{*}(i)\right\rangle=\eta_{i} \mathscr{B}\left(K_{S} \rightarrow i\right), \quad i=\pi^{0} \pi^{0}, \pi^{+} \pi^{-}(\gamma), 3 \pi^{0}, \pi^{0} \pi^{+} \pi^{-}(\gamma)
$$

The experimental knowledge of these $\alpha_{i}$ 's generate directly the contributions to the righthand side in (3.1) while for semileptonic modes we have to work hard theoretically and experimentally to determine this contribution. To show the relative size of the various contributions we show in Table 1 the Standard Model expectations for these quantities ${ }^{1}$.

- the recent data from CPLEAR, KLOE, KTeV and NA48 have led the following determinations (the analysis described in ref. [19] has been updated by using the recent measurements of $K_{L}$ branching ratios from $\mathrm{KTeV}[21]$ and NA48[22])

$$
\begin{aligned}
\alpha_{\pi^{+} \pi^{-}} & =((1.112 \pm 0.013)+i(1.061 \pm 0.014)) \times 10^{-3} \\
\alpha_{\pi^{0} \pi^{0}} & =((0.493 \pm 0.007)+i(0.471 \pm 0.007)) \times 10^{-3} \\
\alpha_{\pi^{+} \pi^{-} \pi^{0}} & =((0 \pm 2)+i(0 \pm 2)) \times 10^{-6} \\
\left|\alpha_{\pi^{0} \pi^{0} \pi^{0}}\right| & <7 \times 10^{-6} \text { at } 95 \% \mathrm{CL} .
\end{aligned}
$$

We stress that this determination does not require the knowledge of the $B_{I}$ 's in (2.4)

\section{CPT in semileptonic decays}

We will discuss the semileptonic decays of neutral kaons without assuming the $\Delta S=\Delta Q$ rule and the CPT symmetry [1], 2, 15].

\footnotetext{
${ }^{1}$ I thank KLOE Coll. and Gino Isidori for helping in evaluating this table
} 
The $\Delta S=\Delta Q$ rule is well supported by experimental data and is naturally accounted for by the Standard Model, where the $\Delta S=-\Delta Q$ transitions are possible only with two effective weak vertices. Explicit calculations in the SM give a suppression factor of order $10^{-6}-10^{-7}$ [23]. Furthermore in any quark model, $\Delta S=-\Delta Q$ transitions can be induced only by operators with dimension higher than 6 and therefore should be suppressed [1, 24]. However large violation of the $\Delta S=\Delta Q$ rule does not conflict with any general principle. We can write [1], 2]

$$
\begin{aligned}
& A\left(K^{0} \rightarrow l^{+} v \pi^{-}\right)=a+b=a(1-y) \\
& A\left(K^{0} \rightarrow l^{-} v \pi^{+}\right)=c+d=a^{*}\left(x_{+}-x_{-}\right)^{*} \\
& A\left(\bar{K}^{0} \rightarrow l^{-} v \pi^{+}\right)=a^{*}-b^{*}=a^{*}(1+y)^{*} \\
& A\left(\bar{K}^{0} \rightarrow l^{+} v \pi^{-}\right)=c^{*}-d^{*}=a\left(x_{+}+x_{-}\right)
\end{aligned}
$$

CPT implies $b=d=0$, CP implies $\mathfrak{I}(a)=\mathfrak{I}(c)=\mathfrak{R}(b)=\mathfrak{R}(d)=0$, T requires real amplitudes and $\Delta S=\Delta Q$ implies $c=d=0$. Then $x_{+}\left(x_{-}\right)$describes the violation of the $\Delta S=\Delta Q$ rule in $C P T$ conserving (violating) decay amplitudes, and $y$ parametrizes $C P T$ violation for $\Delta S=\Delta Q$ transitions.Then

$$
\begin{gathered}
A_{S, L}=\frac{\Gamma_{S, L}^{l^{+}}-\Gamma_{S, L}^{l^{-}}}{\Gamma_{S, L}^{l^{+}}+\Gamma_{S, L}^{l^{-}}}=2 \Re\left(\varepsilon_{S, L}\right)+2 \Re\left(\frac{b}{a}\right) \mp 2 \Re\left(\frac{d^{*}}{a}\right) \\
A_{S}-A_{L} \propto \Re(\delta), \Re\left(d^{*}\right)
\end{gathered}
$$

Thus a non-vanishing value of the difference $A_{S}-A_{L}$ would be an evidence of CPT violation, either in the mass matrix or in the $\Delta S=-\Delta Q$ amplitudes ( $\delta$ and $d^{*} / a$ cannot be disentangled by semileptonic decays alone). The sum $A_{S}+A_{L}$ has CPT-conserving $\left(\Re\left(\varepsilon_{M}\right)\right)$ and CPT-violating $(\Re(b / a))$ contributions that cannot be disentangled.

Taking advantage of their tagged $K^{0}\left(\bar{K}^{0}\right)$ - beams CPLEAR has measured $\mathfrak{I}\left(x_{+}\right), \mathfrak{R}\left(x_{-}\right), \mathfrak{I}(\boldsymbol{\delta})$, $\Re(\delta)[25,26]$.

\begin{tabular}{l|c|cccc} 
& value & \multicolumn{4}{|c}{ Correlation coefficients } \\
\hline $\mathfrak{R}(\boldsymbol{\delta})$ & $(3.0 \pm 3.4) \times 10^{-4}$ & 1 & & & \\
$\mathfrak{I}(\boldsymbol{\delta})$ & $(-1.5 \pm 2.3) \times 10^{-2}$ & 0.44 & 1 & & \\
$\mathfrak{R}\left(x_{-}\right)$ & $(0.2 \pm 1.3) \times 10^{-2}$ & -0.56 & -0.97 & 1 & \\
$\mathfrak{I}\left(x_{+}\right)$ & $(1.2 \pm 2.2) \times 10^{-2}$ & -0.60 & -0.91 & 0.96 & 1
\end{tabular}

Table 2: CPLEAR determination [25]

These determinations have been improved in Ref.[19] by including the information $A_{S}-A_{L}=$ $4\left[\Re(\delta)+\Re\left(x_{-}\right)\right]$, where $A_{L, S}$, are the $K_{L}$ and $K_{S}$ semileptonic charge asymmetries, respectively from the PDG [5] and KLOE [27]: $A_{S}-A_{L}=(-2 \pm 10) \times 10^{-3}$. The results, referred to as the $K_{\ell 3}$ average, are given in Table 3 


\begin{tabular}{l|c|ccccc} 
& value & \multicolumn{5}{|c}{ Correlation coefficients } \\
\hline $\mathfrak{R}(\boldsymbol{\delta})$ & $(3.4 \pm 2.8) \times 10^{-4}$ & 1 & & & & \\
$\mathfrak{I}(\boldsymbol{\delta})$ & $(-1.0 \pm 0.7) \times 10^{-2}$ & -0.27 & 1 & & & \\
$\mathfrak{R}\left(x_{-}\right)$ & $(-0.07 \pm 0.25) \times 10^{-2}$ & -0.23 & -0.58 & 1 & & \\
$\mathfrak{I}\left(x_{+}\right)$ & $(0.8 \pm 0.7) \times 10^{-2}$ & -0.35 & -0.12 & 0.57 & 1 & \\
$A_{S}+A_{L}$ & $(0.5 \pm 1.0) \times 10^{-2}$ & -0.12 & -0.62 & 0.99 & 0.54 & 1
\end{tabular}

Table 3: Determination after KLOE measurements [19]

The value $A_{S}+A_{L}$ in Tab. 3 can be directly included in the semileptonic contributions to the Bell Steinberger relations in (3.1)

$$
\begin{aligned}
\sum_{\pi \ell v}\left\langle\mathscr{A}_{L}(\pi \ell v) \mathscr{A}_{S}^{*}(\pi \ell v)\right\rangle & =2 \Gamma\left(K_{L} \rightarrow \pi \ell v\right)\left(\mathfrak{R}(\varepsilon)-\mathfrak{R}(y)-i\left(\mathfrak{I}\left(x_{+}\right)+\mathfrak{I}(\delta)\right)\right) \\
& =2 \Gamma\left(K_{L} \rightarrow \pi \ell v\right)\left(\left(A_{S}+A_{L}\right) / 4-i\left(\mathfrak{I}\left(x_{+}\right)+\mathfrak{I}(\delta)\right)\right) .
\end{aligned}
$$

So that now defining

$$
\alpha_{\pi \ell v} \equiv \frac{1}{\Gamma_{S}} \sum_{\pi \ell v}\left\langle\mathscr{A}_{L}(\pi \ell v) \mathscr{A}_{S}^{*}(\pi \ell v)\right\rangle+2 i \frac{\tau_{K_{S}}}{\tau_{K_{L}}} \mathscr{B}\left(K_{L} \rightarrow \pi \ell v\right) \mathfrak{I}(\delta)
$$

we find:

$$
\alpha_{\pi \ell v}=((0.3 \pm 0.6)+(-1.8 \pm 1.8 i)) \times 10^{-5} .
$$

This value can be used in the Bell Steinberger relations in (3.1) to determine $\mathfrak{R}(\varepsilon)$ and $\mathfrak{I}(\delta)$ [19] :

$$
\text { KLOE } \mathfrak{R}(\varepsilon)=(159.6 \pm 1.3) \times 10^{-5}, \quad \mathfrak{I}(\delta)=(0.4 \pm 2.1) \times 10^{-5},
$$

improving CPLEAR [25, 26],

$$
\text { CPLEAR } \quad \mathfrak{R}(\varepsilon)=(164.9 \pm 2.5) \times 10^{-5}, \quad \mathfrak{I}(\boldsymbol{\delta})=(2.4 \pm 5.0) \times 10^{-5} .
$$

Since

$$
\delta=\frac{i\left(m_{K^{0}}-m_{\bar{K}^{0}}\right)+\frac{1}{2}\left(\Gamma_{K^{0}}-\Gamma_{\bar{K}^{0}}\right)}{\Gamma_{S}-\Gamma_{L}} \cos \phi_{S W} e^{i \phi_{S W}}[1+\mathscr{O}(\varepsilon)]
$$

KLOE gets

$$
-5.3 \times 10^{-19} \mathrm{GeV}<m_{K^{0}}-m_{\bar{K}^{0}}<6.3 \times 10^{-19} \mathrm{GeV} \quad \text { at } 95 \% \mathrm{CL},
$$

improving CPLEAR result, $\left|m_{K^{0}}-m_{\bar{K}^{0}}\right|<12.7 \times 10^{-19} \mathrm{GeV}$ at $90 \% \mathrm{CL}$.

\section{Outlook}

A further improvement can be obtained by including the $T$-violating asymmetry measurement from CPLEAR without any assumption on the $\Delta S=-\Delta Q$ transitions, i.e. relaxing the constraint on $\mathfrak{R}\left(x_{-}\right)=\mathfrak{I}\left(x_{+}\right)=0$ imposed in the original paper [28] to evaluate the $\mathfrak{R}(\Delta)$ and $\mathfrak{I}(\Delta)$ parameters. 


\section{Acknowledgments}

I thank the organizers of KAON07 for the conference and the nice invitation, Mario Antonelli and Gino Isidori for conversations and collaboration. This work has been supported in part by the European Commission (EC) RTN FLAVIAnet under Contract No. MRTN-CT-2006-035482 and by the Italian Ministry of Education and Research (MIUR), project 2005-023102.

\section{References}

[1] L. Maiani, "CP And CPT Violation In Neutral Kaon Decays," L. . Maiani, G. . Pancheri and N. . Paver,"The Second Daphne Physics Handbook. Vol. 1, 2,"

[2] G. D’Ambrosio, G. Isidori and A. Pugliese, arXiv:hep-ph/9411389.

[3] O. W. Greenberg, arXiv:hep-ph/0309309; and references therein.

[4] G. D’ Ambrosio and G. Isidori Int. J. Mod. Phys. A 13 (1998) 1 [arXiv:hep-ph/9611284].

[5] W. M. Yao et al. [Particle Data Group], J. Phys. G 33 (2006) 1.

[6] R. Bluhm, arXiv:hep-ph/0308281, and references therein.

[7] S. R. Coleman and S. L. Glashow, Phys. Rev. D 59 (1999) 116008 [arXiv:hep-ph/9812418].

[8] V. A. Kostelecky and S. Samuel, Nucl. Phys. B 336 (1990) 263; V. A. Kostelecky and R. Potting, Nucl. Phys. B 359 (1991) 545.

[9] A. Jenkins, Phys. Rev. D 69 (2004) 105007 [arXiv:hep-th/0311127]; A. A. Andrianov and R. Soldati, Phys. Lett. B 435 (1998) 449 [arXiv:hep-ph/9804448]; and references therein.

[10] G. Barenboim and J. Lykken, Phys. Lett. B 554, 73 (2003) [arXiv:hep-ph/0210411].

[11] O. W. Greenberg, Phys. Lett. B 567 (2003) 179 [arXiv:hep-ph/0305276]; L. Cappiello and G. D'Ambrosio, arXiv:hep-th/0405276.

[12] L. Cappiello and G. D’Ambrosio, JHEP 0407 (2004) 002 [arXiv:hep-th/0405276].

[13] R. Peccei, in "Results and perspectives in particle

[14] J. R. Ellis, N. E. Mavromatos and D. V. Nanopoulos, Phys. Lett. B 293 (1992) 142 [arXiv:hep-ph/9207268].

[15] M. S. Sozzi and I. Mannelli, Riv. Nuovo Cim. 26 (2003) 1 [arXiv:hep-ex/0312015].

[16] S.W. Hawking, Phys. Rev. D14 (1975) 2460.

[17] P. Huet and M. E. Peskin, Nucl. Phys. B 434 (1995) 3 [arXiv:hep-ph/9403257].

[18] J. S. Bell and J. Steinberger, in Oxford International Symposium Conference on elementary particles, September 1965, 195-208, 221-222

[19] F. Ambrosino et al. [KLOE Collaboration], JHEP 0612 (2006) 011 [arXiv:hep-ex/0610034].

[20] A. Alavi-Harati et al. [KTeV Collaboration], Phys. Rev. D 67 (2003) 012005 [arXiv:hep-ex/0208007].

[21] T., Alexopoulos et al. [KTeV Collaboration], Phys. Rev. D 70, 092006 (1998).

[22] A. Lai et al. [NA48 Collaboration], Phys. Lett. B 645, 26 (2007); A. Lai et al. [NA48 Collaboration], Phys. Lett. B 602, 41 (2004). 
[23] C.O. Dib and B.Guberina, Phys. Lett. B 255 (1991) 113; M. Luke, Phys. Lett. B 256 (1991) 265.

[24] P. Franzini, arXiv:hep-ex/0401031.

[25] A. Angelopoulos et al. [CPLEAR Collaboration], Phys. Lett. B 444, 52 (1998).

[26] A. Angelopoulos et al. [CPLEAR Collaboration], Phys. Rept. 374 (2003) 165.

[27] F. Ambrosino et al. [KLOE Collaboration], Phys. Lett. B 636 (2006) 173 [arXiv:hep-ex/0601026].

[28] A. Angelopoulos et al. [CPLEAR Collaboration], Phys. Lett. B 444 (1998) 43. 